\title{
Coupled Quantum Dots - Spatial Correlations between Interacting Carriers
}

\author{
B. SZAFRAN
}

Faculty of Physics and Applied Computer Science

AGH University of Science and Technology

al. Mickiewicza 30, 30-059 Kraków, Poland

\begin{abstract}
We review results of our modeling of excitons and excitonic trions confined in vertically stacked InGaAs/GaAs self-assembled quantum dots. Electrons and holes in double quantum dots are much more significantly correlated than in a single dot. For that reason our modeling was based on simple confinement potentials that allow for an exact diagonalization of the resulting two- and three-particle Hamiltonians with a precise account for the relative electron and hole localization along the stack. We studied the optical signatures of the coupling in context of the photoluminescence experiments performed in the external electric field. The calculations predicted prior to the experiment the mechanism of the exciton and negative trion dissociation by electron removal from the dot occupied by the hole. We discuss the competition between the tunnel and the electrostatic interdot couplings. Effects of the non-perfect alignment of the dots as well as stacks containing more than two dots are also discussed.
\end{abstract}

PACS numbers: 73.21.La, 73.43.-f, 71.10.Pm, 71.35.-y

\section{Introduction}

Epitaxial growth of strained InAs layers on GaAs substrate results in formation of partly alloyed self-assembled quantum dots. The strain field favors nucleation of subsequent dots above the pre-existing ones, which leads to growth of vertical stacks of dots [1-3]. Photoluminescence spectra of large dot ensembles exhibit redshifts of the spectral lines with decreasing thickness of the tunnel barrier [1-3]. The redshifts, also observed in function of the number of dots forming the stack [2], were identified as due to the electron tunnel coupling, and thus provided an optical evidence for formation of electron artificial molecular orbitals extended over several dots (several artificial atoms). The early experiments on stacks of dots [1-3] were performed in the absence of an external electric field. In order to track the redshifts with decreasing barrier thickness a number of measurements were performed [1-3] each time on a different sample. An alternative solution 
to changing samples is an application of an external vertically-oriented electric field, since it tunes the character of the electron and the hole orbitals between molecular (extended over two dots) and ionic (localized in a single dot) types for a fixed interdot barrier thickness. Such measurements for a single pair of dots separated of the entire ensemble have been realized only relatively recently $[4,5]$. In the present paper we review our work on interacting electrons and holes in vertically stacked InGaAs/GaAs self-assembled quantum dots [6-12] in context of the photoluminescence experiments performed in external electric field.

A typical InGaAs self-assembled quantum dot has a vertical height of about $4 \mathrm{~nm}$ and lateral radius of $10-12 \mathrm{~nm}$. The lateral size is roughly equal or smaller than the bulk exciton size (exciton radius is $\approx 10 \mathrm{~nm}$ in GaAs, and $\approx 30 \mathrm{~nm}$ in InAs). For that reason wave functions of the carriers confined in a single quantum dot are only slightly more localized by the electron-hole interaction. It is commonly accepted that in the modeling of a single quantum dot the single-particle effects are crucial while the electron-hole interaction is of a secondary importance. This methodology was also applied in theoretical papers $[13,14]$ of 2002 , addressing the electric-field effect on a double quantum dot. The single-particle effects were described $[13,14]$ with a special care, but the electron-hole interaction was neglected [13] or treated in an approximate manner [14]. Both calculations $[13,14]$ assumed the total exciton wave function in form of a separable product of single-electron and single-hole wave functions. As a result it was predicted [13, 14] that the ground-state exciton line in the photoluminescence spectrum possesses a cusp when the localization of the hole is changed by the electric field. The cusp was announced as an anomaly compared to the parabolic field dependence of the ground-state energy obtained [15] for a single dot in perturbation theory of a non-degenerate ground state.

The experiments published $[4,5]$ in 2005 established that the exciton dissociation appears through an avoided crossing of photoluminescence lines with a pronounced modulation of recombination probabilities with the external electric field. No cusp as predicted by the modeling applying a separable wave function was observed. Our early work on tunnel coupling of interacting electron-hole pair [6] indicated that for identical dots outside the strong coupling limit the total exciton wave function is essentially entangled and not separable. Based on this inspiration we proposed an exactly-solvable numerical approach [7] to treat the exciton in a double quantum dot with a confinement potential that was realistic enough to model the actual measurements and simple enough to allow for a full account for the electron-hole correlation. The modeling [7] predicted a correct mechanism of the exciton dissociation by the electric field. It is important to note that our work [7] was submitted prior to the experimental papers $[4,5]$. For the last three years the photoluminescence experiments in the external electric field provided a rich material [16-22] on the tunnel coupling and the electron-hole interaction for the exciton and exciton complexes in double dots. In particular our 
prediction [7] of the mechanism of the exciton trion dissociation by the field was precisely confirmed by the experiment described in Ref. [18].

The paper is organized as follows: In Sect. 2 we briefly describe the theory that we used. In Sect. 3 we discuss the electron and hole localization in a pair of identical dots without the electric field. The effect of the field for identical and non-identical dots is described in Sect. 4. Section 5 contains the results for the trion dissociation. Section 6 reviews our recent results for the rotated in-plane electric field for non-perfectly aligned dots and Sect. 7 provides results for a stacks of three dots in vertical electric field. Section 8 contains the summary and conclusions.

\section{Theory}

In our modeling [6-12] we used the following Hamiltonian for the electronhole pair:

$$
\begin{aligned}
H= & -\frac{\hbar^{2}}{2 m_{\mathrm{e}}} \nabla_{\mathrm{e}}^{2}-\frac{\hbar^{2}}{2 m_{\mathrm{h}}} \nabla_{\mathrm{h}}^{2}+V_{\mathrm{e}}\left(\boldsymbol{r}_{\mathrm{e}}\right)+V_{\mathrm{h}}\left(\boldsymbol{r}_{\mathrm{h}}\right) \\
& -\frac{e^{2}}{4 \pi \epsilon \epsilon_{0}\left|\boldsymbol{r}_{\mathrm{e}}-\boldsymbol{r}_{\mathrm{h}}\right|}-e \boldsymbol{F}\left(\boldsymbol{z}_{\mathrm{e}}-\boldsymbol{z}_{\mathrm{h}}\right),
\end{aligned}
$$

where $\boldsymbol{r}_{\mathrm{e}}, \boldsymbol{r}_{\mathrm{h}}$ are the electron and the hole coordinates, $V_{\mathrm{e}}, V_{\mathrm{h}}$ stand for the confinement potential for the electron and the hole, and $\boldsymbol{F}$ is the electric field oriented along the growth direction (see Fig. 1). The material parameters and potential well depth for the electrons and holes were adopted for the dot composed of $\mathrm{In}_{0.66} \mathrm{Ga}_{0.34}$ As alloy embedded in GaAs. We used effective masses $m_{\mathrm{e}}=0.037 m_{0}$ for the electron and $m_{\mathrm{h}}=0.45 m_{0}$ for the hole, as well as the dielectric constant of $\epsilon=12.5$. The model Hamiltonian (1) is written in the single-band heavyhole approximation, which is justified by a small height of a single dot* for which the contribution of the light hole to the lowest-energy exciton states is negligible. We also neglect the electron-hole exchange interaction. The electron-hole exchange energy was recently estimated by application of the transverse magnetic field which mixes dark and bright exciton states [21]. The estimated value [21] of $0.24 \mathrm{meV}$ is small at the energy scale of the avoided crossings observed at the exciton dissociation by the field.

The specific confinement potentials that we used for the electron and hole as well as the treatment of the two-body problem evolved as we studied various aspects of the system. In the first paper [6] (Sect. 3) we were interested in the electron-hole correlation for a pair of identical dots in the absence of the electric field. In this work [6] we used cylindrical quantum wells of a Gaussian profile

${ }^{*}$ Confinement energy of a light hole is much larger than the one of the heavy hole. The ground-state energy for a particle of an effective mass $m$ in an infinite quantum one-dimensional quantum well of width $L$ is given by $E=\hbar^{2} \pi^{2} / 2 m L^{2}$. For $L=5 \mathrm{~nm}$ (which roughly corresponds to the height of self-assembled dots) the ground-state energy of a heavy hole of mass $m=0.5 m_{0}$ is $30 \mathrm{meV}$ (from the top of the valence band), while for a light hole of mass $m=0.05 m_{0}$ the ground-state energy is as large as $300 \mathrm{meV}$. 


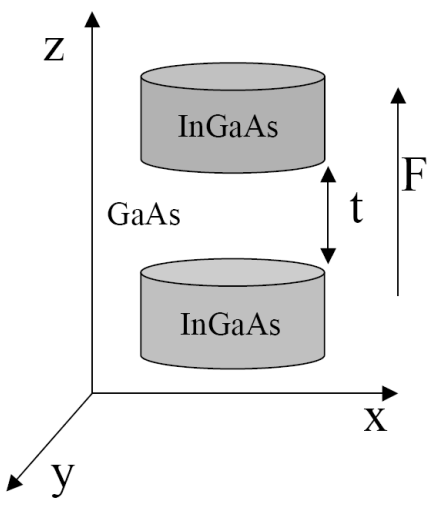

Fig. 1. Schematic drawing of the model system of two vertically stacked dots separated by a tunnel barrier of thickness $t$ with electric field oriented vertically.

for the confinement model. The exciton problem was solved with a variational method. In the trial wave function we assumed that not only the total angular momentum but also the single-electron and single-hole angular momenta are separately defined. This approximation is justified by the strong confinement in each dot and consequently by large energy spacings for single-particle states of different angular momenta. On the other hand, the energy spacing between states of vertical quantization may be arbitrarily small. For that reason the electron-hole correlation mainly appears in localization of the electron and hole in the same or separate dots. The trial wave function [6] was designed to be particularly flexible for description of vertical localization effects for both the particles.

In Ref. [7] (Sect. 4 and partially 5) we studied the effect of the electric field oriented in the vertical direction. In order to correctly describe the reaction of the electron and the hole to the field we replaced the Gaussian confinement model by a more realistic square quantum well potential. In the lateral direction (perpendicular to the field) we introduced the harmonic oscillator potential with the same energy $\hbar \omega$ for the electron and for the hole

$$
V_{\mathrm{e}}\left(\rho_{\mathrm{e}}\right)+V_{\mathrm{h}}\left(\rho_{\mathrm{h}}\right)=\frac{\omega^{2}}{2}\left(m_{\mathrm{h}} \rho_{\mathrm{h}}^{2}+m_{\mathrm{e}} \rho_{\mathrm{e}}^{2}\right) .
$$

This model potential allowed us to separate out the lateral center of mass motion and get rid of two of the total six coordinates describing the problem

$$
\Psi\left(\boldsymbol{r}_{\mathrm{e}}, \boldsymbol{r}_{\mathrm{h}}\right)=R\left(x_{\mathrm{cm}}, y_{\mathrm{cm}}\right) \chi\left(x_{\mathrm{eh}}, y_{\mathrm{eh}} z_{\mathrm{e}}, z_{\mathrm{h}}\right),
$$

where $R\left(x_{\mathrm{cm}}, y_{\mathrm{cm}}\right)$ is the wave function of the lateral center of mass motion, and $x_{\mathrm{eh}}=x_{\mathrm{e}}-x_{\mathrm{h}}, y_{\mathrm{eh}}=y_{\mathrm{e}}-y_{\mathrm{h}}$. Furthermore, we used the cylindrical symmetry of the relative eigenproblem

$$
\chi\left(x_{\mathrm{eh}}, y_{\mathrm{eh}} z_{\mathrm{e}}, z_{\mathrm{h}}\right)=\exp \left(\mathrm{i} L \phi_{\mathrm{eh}}\right) \psi_{L}\left(\rho_{\mathrm{eh}}, z_{\mathrm{e}}, z_{\mathrm{h}}\right),
$$

where $L$ is the angular momentum carried by the relative electron-hole motion. The remaining problem defined in a space of only three coordinates (the lateral 
relative electron-hole distance $\rho_{\mathrm{eh}}$ and vertical positions of both the electron and the hole: $z_{\mathrm{e}}, z_{\mathrm{h}}$ ) was treated with a finite difference technique, i.e. without assumption of any variational wave function. Therefore the electron-hole correlation in this simple confinement potential was treated exactly. Assumption of the equal confinement energy of lateral harmonic-oscillator confinement for the electron and the hole implied that the radius of the hole localization was about 3 times smaller than the one of electron $\left(r_{p}=\sqrt{\hbar / m_{p} \omega}, p=\mathrm{e}, \mathrm{h}\right)$. In real dots the hole is indeed more strongly localized due to its larger mass, but the model potential overestimates the increased hole localization significantly. In a more realistic quantum disk model of confinement [14] the lateral localization radius for typical size and depth of the quantum well is about 1.2-1.5 times smaller than the one for the electron. The overestimated localization of the hole in the discussed model [7] does not introduce any qualitative consequences for the electric field effect applied in the vertical direction — as confirmed by our recent studies $[10,12,11]$ where we used the three-dimensional quantum wells for modeling the confinement potential (Sects. 6 and 7). The recent results were obtained with the configuration interaction approach in which the exciton wave function is diagonalized in basis of products of single-electron and single-hole wave functions. This is a brute force technique that provides exact results provided that the basis is large enough. With the computational power of present desktop PCs the convergence of the method for a pair of carriers is obtained without any significant difficulties for up to four quantum dots forming the stack [11].

\section{Electron and hole localization in a pair of identical dots in the absence of the external field}

The dots that are actually produced are never identical [1, 2]. In the Stranski-Krastanov growth mode for the same nominal number of InAs monolayers deposited for each layer of dots, the size of the quantum dots tends to increase along the stack $[1,2]$. Nevertheless, in the experiments $[4,5]$ hybridization of atomic (single-dot) into molecular (extended) orbitals occurs when the electric field compensates for the unequal confinement. The transfer of the electron or the hole from one dot to the other occurs when the electric field makes the dots effectively of similar depth for the carriers. The ideal case of exactly identical dots was studied in Ref. [6]. Results of this paper are discussed in this section. We considered nonidentical dots in later papers [7, 10-12] (see Sects. 5, 6, and 7).

Figure 2a shows the single-electron and single-hole energy levels (the results of this section come from Ref. [6]) in function of the spacer thickness $t$. Splitting of the electron energy levels related to formation of binding and antibinding molecular orbitals is visible already for $t=12 \mathrm{~nm}$. The hole, which is about 10 times heavier than the electron, forms molecular orbitals only for $t<5 \mathrm{~nm}$, and the energy splitting of the binding and antibinding levels is much smaller than for the electron.

In Fig. 2b exciton energy levels of zero total angular momentum are presented (recombination is forbidden for states in which the envelope wave function 


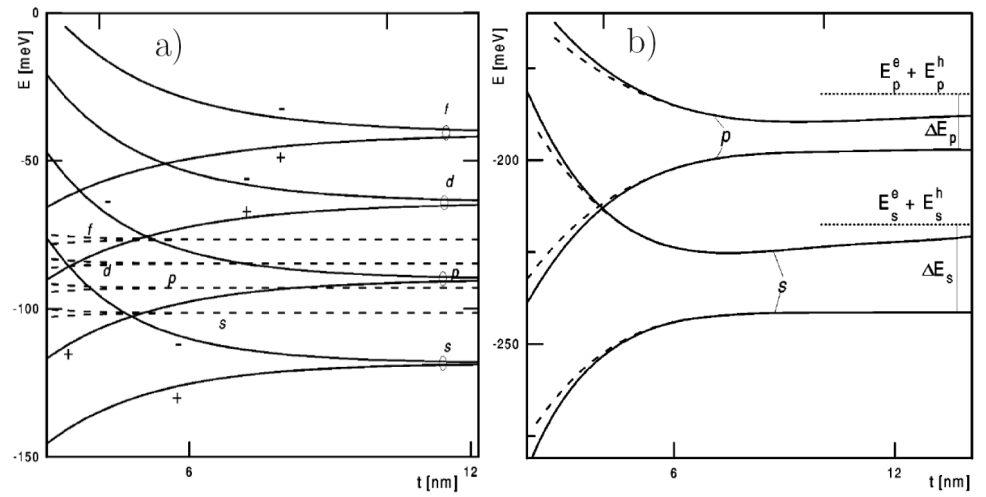

Fig. 2. (a) Energy levels of a single electron (solid lines) and a single hole in function of the barrier thickness for a pair of identical Gaussian dots. Labels $s, p, d$ and $f$ correspond to angular momenta $l=0,1,2$, and 3 . By + and - we denote even and odd parity (binding and antibinding orbitals). (b) Zero-angular momentum energy levels for electron-hole pair. States marked by $s(p)$ correspond to states constructed of single-electron and single-hole angular momenta equal to 0 (1). Solid (dashed) lines show energy levels of the even (odd) parity states. Reprinted figures with permission from Ref. [6]. Copyright (2001) by the American Physical Society.

carries a non-zero angular momentum). Let us focus on the four energy levels marked by $s$, in which additionally both the electron and hole angular momentum is zero. By solid (dashed) lines we plot the energy of states of the even (odd) total parity. In the large $t$ limit both the ground-state energy level and the first excited energy level are twofold degenerate. For non-interacting carriers a fourfold degeneracy should be expected - with respect to the parities of both the particles separately. For large $t$ the ground-state energy becomes independent of the interdot distance, but the energy of the first excited state grows with $t$. Different behavior of the energies of the ground state and the first excited state with $t$ is due to a different electron and hole distribution in both these states. In the ground state both the particles occupy the same dot, and in the first excited state the hole and the electron occupy different dots.

For $t<8 \mathrm{~nm}$ the ground state starts to go down in the energy, while the excited state starts to go up (see Fig. 2b). This is due to activation of the electron tunnel coupling. The ground state is associated to the binding electron orbital and the excited state to the antibinding orbital. Both the ground state and first excited state remain degenerate for $t>5 \mathrm{~nm}$. Degeneracy of the ground-state energy level and the first excited energy levels is lifted when the hole tunnel coupling appears.

Figure 3 a shows the exciton ground-state wave function plotted for the strong coupling case of $t=2.2 \mathrm{~nm}$. The wave function is plotted in function of the electron and hole vertical positions $\left(z_{\mathrm{e}}, z_{\mathrm{h}}\right.$, corresponding to horizontal and vertical axes, respectively) calculated at the symmetry axis of the system $(x=y=0)$. The 


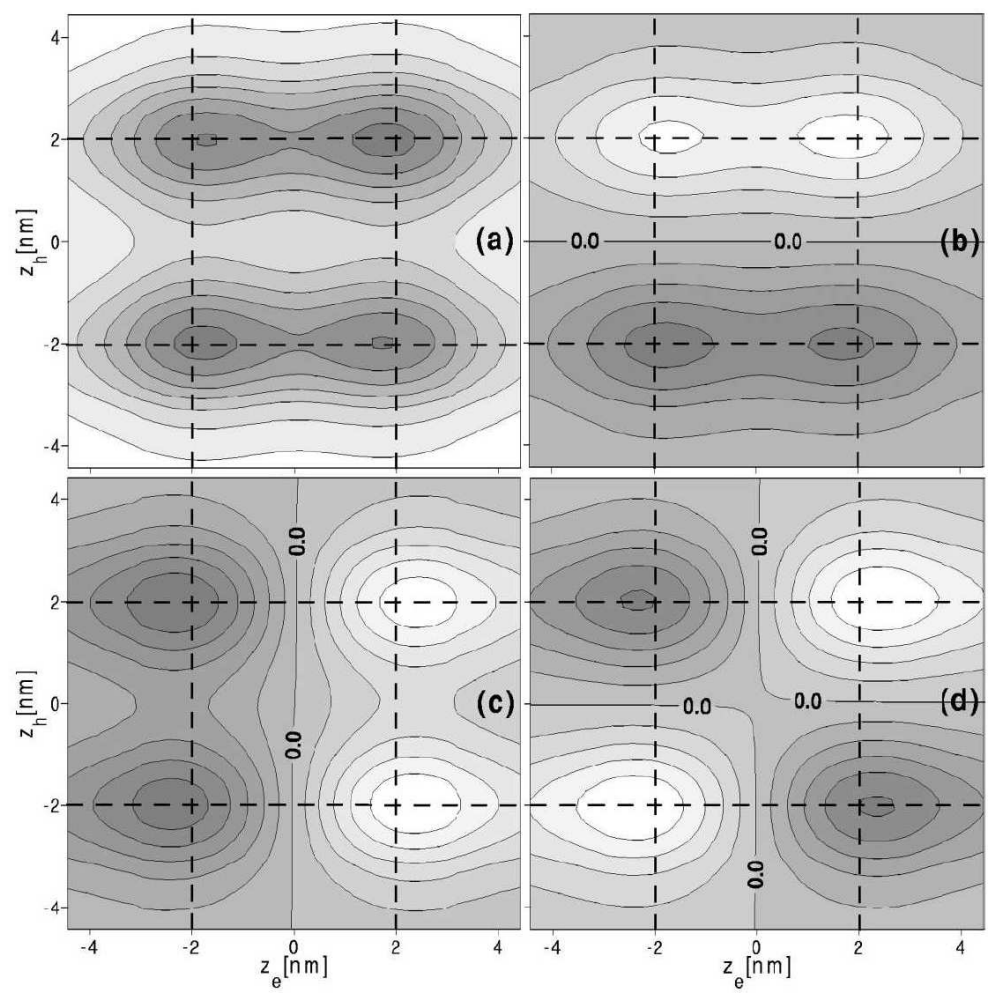

Fig. 3. Exciton wave function $\Psi\left(\boldsymbol{r}_{\mathrm{e}}, \boldsymbol{r}_{\mathrm{h}}\right)$ plotted on the axis of the double dot system $x_{\mathrm{e}}=y_{\mathrm{e}}=x_{\mathrm{h}}=e_{\mathrm{h}}=0$ in function of the vertical coordinates of the electron (horizontal axes) and the hole (vertical axes) for barrier thickness of $t=7 \mathrm{~nm}$. Dashed lines show the positions of the dot centers. Plots $(\mathrm{a}, \mathrm{b}, \mathrm{c}, \mathrm{d})$ correspond to the ground state, first, second and third excited state for the electron and hole angular momenta of $l=0$ (marked by $s$ in Fig. 2b) for $t=2.2 \mathrm{~nm}$. Reprinted figure with permission from Ref. [6]. Copyright (2001) by the American Physical Society.

Hamiltonian eigenstates for two identical dots have a definite total parity. The plot for the ground state in Fig. 3a is exactly symmetric with respect to the simultaneous interchange of the signs of the electron and hole coordinates (i.e. with respect to inversion through the center of the plot). We also notice an approximate symmetry of the ground-state wave function with respect to a separate inversion of the electron or the hole coordinate. The approximate symmetry is due to the fact that in the strong coupling limit the splitting of the binding and antibinding energy levels becomes much larger than the interaction energy ${ }^{\dagger}$. The ground-state

${ }^{\dagger}$ The results of Figs. $2-5$ were obtained for a model in which the electron-hole interaction energy for a single dot equals $24 \mathrm{meV}$. For $t=2.2 \mathrm{~nm}$ the electron energy splitting is of the order of $100 \mathrm{meV}$. The splitting for the hole is of the order of $10 \mathrm{meV}$. It could seem that the antibinding hole state should contribute strongly to the ground state. This is not 


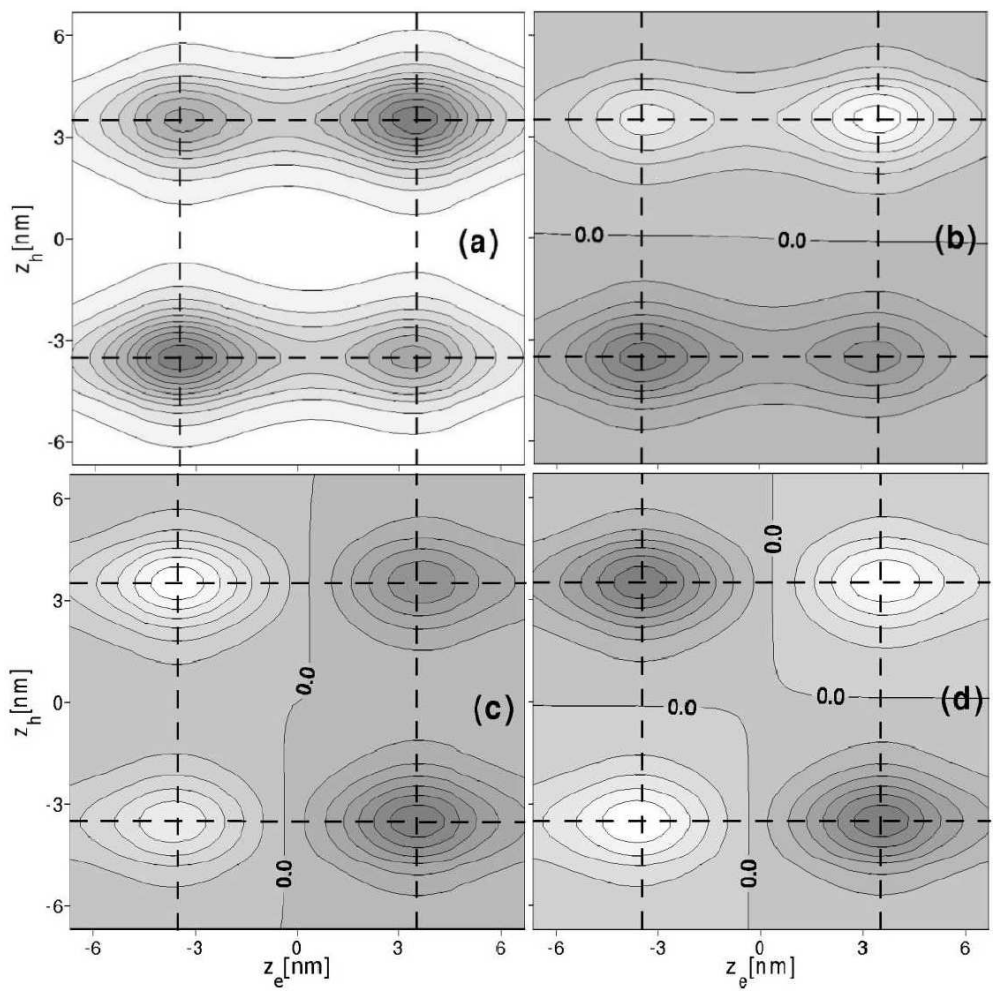

Fig. 4. The same as Fig. 3 but for $t=5.2 \mathrm{~nm}$. Reprinted figure with permission from Ref. [6]. Copyright (2001) by the American Physical Society.

wave function of Fig. 3a has four local maxima near the crossing of the dashed lines which show the position of the centers of the two dots for $z_{\mathrm{e}}$ and $z_{\mathrm{h}}$ coordinates. The maxima at the left-lower and right-upper corners of the plot correspond to the electron and the hole residing in the same dot (coordinates of the electron and hole are either both positive or both negative). The maxima at the other two crossings of the dashed lines correspond to the electron and the hole localized in different dots (coordinates of the electron and the hole have opposite signs). In the first excited state (cf. Fig. 3b) the electron is still in the binding state but the hole is in the antibinding state - see the nodal line of the wave function for $z_{\mathrm{h}}=0$. The first excited state possesses an odd total parity and consequently the wave function plot is exactly antisymmetric with respect to the origin. This wave function is also nearly symmetric with respect to the inversion of the electron

the case, since for the ground-state which is of the even total parity the antibinding hole state can contribute to the ground-state only along with the antibinding electron state. Hence the ground-state can be written as $\Psi=c_{1} \Psi_{\mathrm{eb}}\left(\boldsymbol{r}_{\mathrm{e}}\right) \Psi_{\mathrm{hb}}\left(\boldsymbol{r}_{\mathrm{h}}\right)+c_{2} \Psi_{\mathrm{ea}}\left(\boldsymbol{r}_{\mathrm{e}}\right) \Psi_{\mathrm{ha}}\left(\boldsymbol{r}_{\mathrm{h}}\right)$, where $\Psi_{\mathrm{eb}}, \Psi_{\mathrm{hb}}, \Psi_{\mathrm{ea}}, \Psi_{\mathrm{ha}}$ are the electron-binding, hole-binding, electron-antibinding and hole-antibinding wave functions, respectively. Due to the huge electron-splitting $c_{1} \gg c_{2}$. 


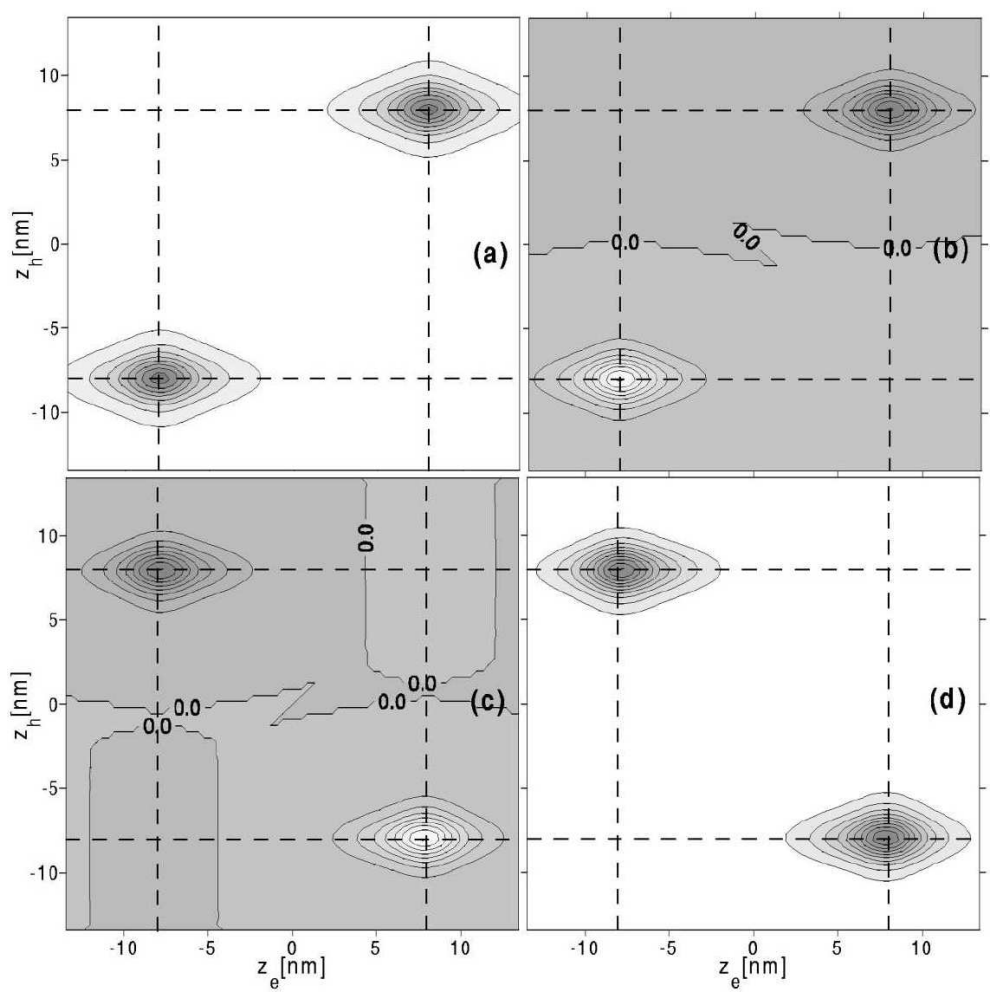

Fig. 5. The same as Fig. 4 but for $t=10.2 \mathrm{~nm}$. Reprinted figure with permission from Ref. [6]. Copyright (2001) by the American Physical Society.

coordinate (reflection through $z_{\mathrm{e}}=0$ line) as well as nearly antisymmetric with respect to the inversion of the hole coordinate (reflection through $z_{\mathrm{h}}=0$ line). In the second excited state (Fig. 3c) the electron occupies an antibinding and the hole a binding one. Both the particles are in the antibinding states in the third excited state (Fig. 3d).

Electron-hole recombination probability of an eigenstate described by wave function $\Psi$ is given by

$$
p=\left|\int \mathrm{d}^{3} \boldsymbol{r}_{\mathrm{e}} \int \mathrm{d}^{3} \boldsymbol{r}_{\mathrm{h}} \Psi\left(\boldsymbol{r}_{\mathrm{e}}, \boldsymbol{r}_{\mathrm{h}}\right) \delta^{3}\left(\boldsymbol{r}_{\mathrm{e}}-\boldsymbol{r}_{\mathrm{h}}\right)\right|^{2} .
$$

The recombination probability vanishes when the total parity of the eigenstate is odd. Therefore for wave functions plotted in Fig. 3, only the ground state (Fig. 3a) and the third excited state (Fig. 3d) can produce the photoluminescence signal provided.

When the interdot distance is increased (see Fig. 4 for $t=5.2 \mathrm{~nm}$ ) the hole tunneling disappears. All the wave functions preserve the symmetry or antisymmetry with respect to the inversion through the origin (total parity is either even or odd). We see that the wave functions remain extended over both the dots only 
in the direction of the horizontal axis (i.e. in function of the electron coordinate only). Moreover, we notice that the probability to find both particles in the same dot is increased in the ground state but decreased in the other even-parity energy level of Fig. 4d.

For even larger distance of $t=10.2 \mathrm{~nm}$ (see Fig. 5) the electron and the hole in the ground state are localized in the same dot (Fig. 5a), while for the excited state of even parity (Fig. 5d) they occupy opposite dots. For the barrier thickness that is so large the wave functions do not exhibit any trace of singleparticle parities (any symmetry or antisymmetry with respect to reflection through $z_{\mathrm{e}}=0$ or $\left.z_{\mathrm{h}}=0\right)$. The single-particle tunnel coupling is completely dominated by the electron-hole interaction. Of the four considered states the recombination is only possible from the ground state whose wave function is plotted in Fig. 5a. In the other ground state (Fig. 5b) recombination is forbidden by the symmetry. In the excited state (Fig. 5c) recombination is forbidden by both symmetry and carrier separation (vanishing wave function at $z_{\mathrm{e}}=z_{\mathrm{h}}$ line). For the wave function plotted in Fig. 5d the recombination is forbidden but not by symmetry-related selection rules but by spatial separation of the carriers. One can imagine that the integration of the recombination probability formula (Eq. (5)) is carried out over the diagonal $\left(z_{\mathrm{e}}=z_{\mathrm{h}}\right)$ line which is empty in Fig. 5d. In modern nomenclature $[4,5]$ the states in which both the particles occupy the same dot are referred to as "direct exciton" states, while the states in which the electron and the hole tend to occupy different dots are called "indirect exciton" states. We will see below that main features of the photoluminescence spectrum in the external electric field result in mixing of direct and indirect exciton states.

Figures 3, 4 and 5 indicate that the electron-hole interaction has a pronounced influence on the relative electron distribution, particularly outside the strong coupling limit. For non-interacting carriers the exciton wave function is a product of single-particle states. Then, for a couple of identical dots in each of the states probability to find the electron and the hole in the same dots is always equal to $50 \%$ independent of the interdot distance which is in a distinct contrast to the results obtained for interacting carriers (see Figs. 3-5). Although for a single self-assembled dot the assumption that the single-particle wave functions are frozen by the strong confinement and not react to the mutual interaction may be a good approximation, it is no longer the case for a pair of coupled dots at least outside the strong coupling limit.

Tendency of the electron and the hole to stay in the opposite or the same dot is increased when the interdot distance is increased. In the ground state of the weak coupling limit the exciton wave function can be approximately written as $\Psi_{\mathrm{d}}\left(\boldsymbol{r}_{\mathrm{e}}, \boldsymbol{r}_{\mathrm{h}}\right)=\phi_{\mathrm{e}}^{1}\left(\boldsymbol{r}_{\mathrm{e}}\right) \phi_{\mathrm{h}}^{1}\left(\boldsymbol{r}_{\mathrm{h}}\right)+\phi_{\mathrm{e}}^{2}\left(\boldsymbol{r}_{\mathrm{e}}\right) \phi_{\mathrm{h}}^{2}\left(\boldsymbol{r}_{\mathrm{h}}\right)$, where $\phi^{1}, \phi^{2}$ are wave function localized in dot 1 and 2 , respectively. The wave function of the indirect excited state of the even total parity and separated carriers is $\Psi_{\mathrm{i}}\left(\boldsymbol{r}_{\mathrm{e}}, \boldsymbol{r}_{\mathrm{h}}\right)=\phi_{\mathrm{e}}^{1}\left(\boldsymbol{r}_{\mathrm{e}}\right) \phi_{\mathrm{h}}^{2}\left(\boldsymbol{r}_{\mathrm{h}}\right)+$ $\phi_{\mathrm{e}}^{2}\left(\boldsymbol{r}_{\mathrm{e}}\right) \phi_{\mathrm{h}}^{1}\left(\boldsymbol{r}_{\mathrm{h}}\right)$. Both wave functions $\Psi_{\mathrm{i}}$ and $\Psi_{\mathrm{d}}$ are entangled and consequently none 
of them can be correctly described by a single product of wave function as assumed by previous modeling $[13,14]$. For separable products on non-interacting states the diagonal $z_{\mathrm{e}}=z_{\mathrm{h}}$ and antidiagonal $z_{\mathrm{e}}=-z_{\mathrm{h}}$ lines in diagrams of Figs. $3-5$ are exactly equivalent. The near equivalence of these lines is only obtained for the strong coupling limit (Fig. 3). The domination of the diagonal or antidiagonal on these diagrams is a signature of the entanglement of the electron and hole states.

Results discussed in this section indicate that the electron-hole interaction and the resulting correlation in the localization of both the particles are of a significant importance for the system and should be accounted for in the calculations describing the electric field effects.

\section{Exciton spectrum of a double dot in the external electric field}

Results of this section come from the exactly solvable numerical model of Ref. [7] (see Sect. 2). The exciton spectrum for a pair of strongly coupled identical dots separated by a barrier of width $t=2 \mathrm{~nm}$ and an electric field applied in the growth direction is plotted in Fig. 6a. A positive electric field tends to transfer the electron to the left dot and the hole to the right one. Radii of the dots placed on the energy levels in Fig. 6 are proportional to the recombination probability. According to the discussion of the previous section for the strong coupling case of $t=2 \mathrm{~nm}$ the hole tunneling is already present and the states possess an approximate singleparticle parities. In the first excited state at $F=0$ the hole is in the antibinding state of the odd parity, so the recombination from this state is forbidden. When the electric field is applied the parities are no longer conserved and recombination can also be obtained from the first excited state (see the inset to Fig. 6a). The electric field mixes the odd and even parity energy levels of the hole producing states in which the hole is totally localized in the right dot (in the ground state) or in the left dot (in the excited state marked by $b_{l}$ in Fig. 6a). At non-zero field both the states produce luminescence signal since the electron (in a binding orbital) is present in both the dots. In the ground state the recombination probability vanishes at larger field when the electron becomes localized in the left dot. In the high $F$ limit in the state corresponding to the $b_{l}$ energy level also the electron occupies the left dot. Consequently the $b_{l}$ level stays optically active. At high field it is accompanied by another bright energy level marked by $b_{r}$ in Fig. 6a with both particles localized in the right dot. When the electric field completely removes the electron tunnel coupling these two energy levels become degenerate. This degeneracy is obtained for $F \approx 100 \mathrm{kV} / \mathrm{cm}$ in case of larger barrier thickness of $t=4 \mathrm{~nm}$ - see the spectrum of Fig. $6 \mathrm{~b}$.

In the absence of the field for $t=2$ and $4 \mathrm{~nm}$ (see Fig. 6a and b) the recombination is allowed not only from the ground state but also from the third excited state. For the intermediate coupling case of $t=7 \mathrm{~nm}$ (see Fig. 6c) at $F=0$ the recombination is only possible from the ground state since in the third excited state the electron and the hole are already separated — localized in different dots 

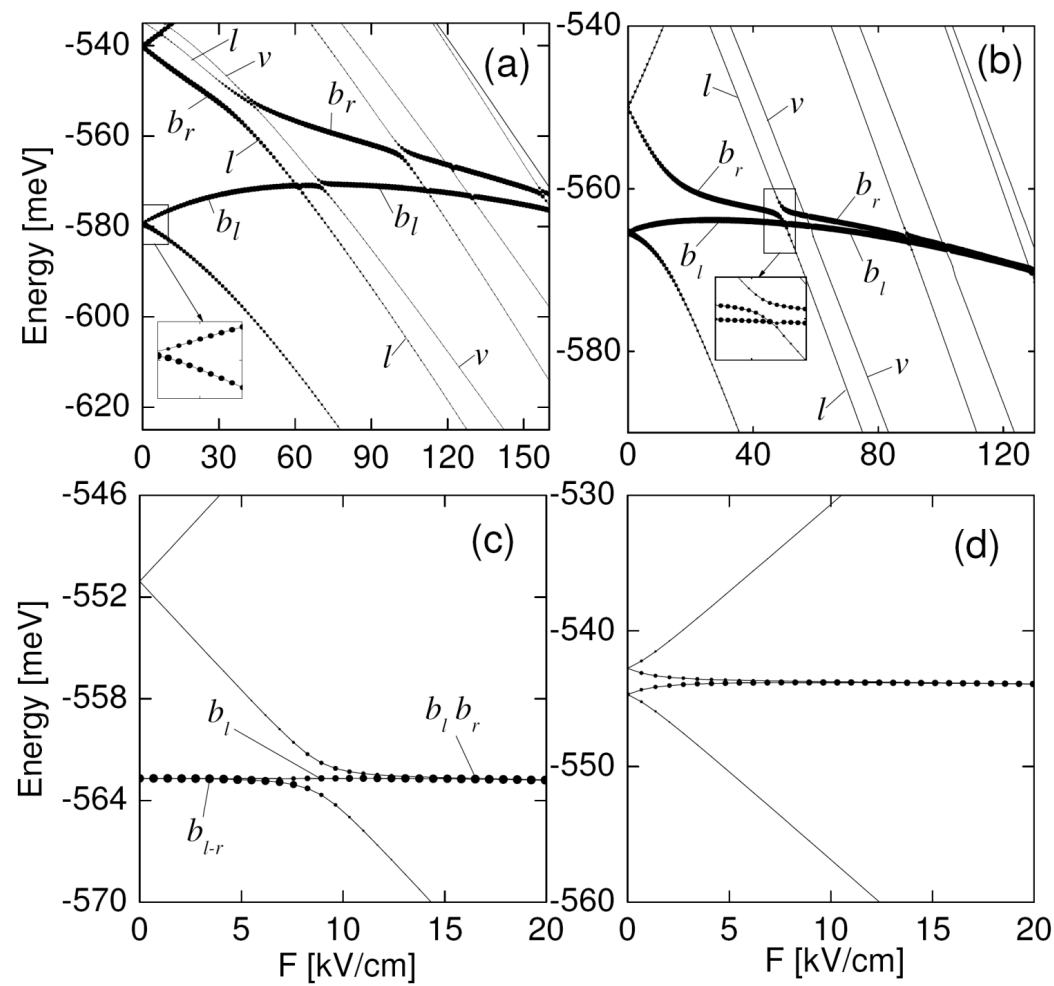

Fig. 6. Energy spectrum of the electron hole pair in a pair of identical dots with the electric field oriented in the growth direction. Positive $F$ tends to transfer the hole to the right (towards increasing $z$ ) and the electron to the left (towards decreasing $z$ ). Radius of the dots is proportional to the recombination probability. Plots (a), (b) and (c) correspond to the barrier thickness of $t=2,4$ and $7 \mathrm{~nm}$, respectively. $b_{l}$ and $b_{r}$ correspond to the direct exciton energy levels in which the hole is localized in the left and right dot, respectively. In (d) parameters are the same as in (c) but the electronhole recombination is switched off. Reprinted figure with permission from Ref. [7]. Copyright (2005) by the American Physical Society.

(this is the indirect exciton state). Evolution of the exciton wave function with the field is plotted in Fig. 7 for the four energy levels of Fig. 6c. Higher plots correspond to higher energies and columns from left to right to increasing electric field. When the electric field is switched on (compare $F=0$ and $F=5 \mathrm{kV} / \mathrm{cm}$ parts) in the ground state the electron and the hole start to occupy rather the right dot and in the first excited state rather the left dot. Both these states correspond to the "direct exciton" localization. The two higher energy levels correspond to the "indirect exciton" states each one associated to a different dot. Figure 6c shows that the ground state does not react to a small $F$ since the intrinsic dipole moment of the system in which the electron and the hole occupy the same dot 
is zero. However, this is not the case for the excited state in which the electron and the hole occupy different dots. The degeneracy of the excited state is split by the external field. The energy level of the state with the electric dipole moment parallel to the field (hole in the right dot, electron in the left dot) decreases with $F$, and the other state with the dipole moment antiparallel to the field increases with $F$. Near $F=7.5 \mathrm{kV} / \mathrm{cm}$ we see an avoided crossing of the indirect exciton energy level and the ground-state energy level. In both anticrossing energy levels the hole is localized in the right dot. At the avoided crossing the localization of the electron in the ground state is modified: the electron is transferred to the left dot. The $b_{l}$ energy level with both particles in the left dot goes unchanged through the center of the avoided crossing. The corresponding state is orthogonal to both interacting states by vanishing overlap of the hole wave functions.

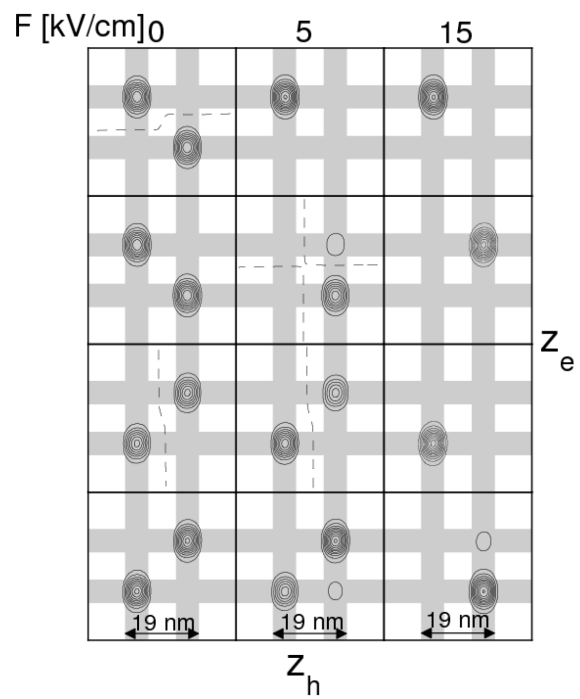

Fig. 7. The exciton wave function of the ground-state (lowest parts) and three lowestenergy excitations (the higher energy the higher plot) corresponding to energy spectrum of Fig. 6c calculated at the axis of the double-dot system in function of the hole and the electron vertical coordinates for various electric fields $F$. The shaded area indicate the region of the potential wells. The dashed lines show the nodal surfaces. Reprinted figure with permission from Ref. [7]. Copyright (2005) by the American Physical Society.

Wave functions plots presented in Fig. 7 for $F=5 \mathrm{kV} / \mathrm{cm}$ indicate a mixing of the ground state and the indirect exciton state with the dipole moment aligned with the field. In the ground state we notice an admixture of the indirect localization with the hole in the right dot and the electron in the left one (see the single contour in the right lower part of the lowest part of Fig. 7). In the indirect state (third part from below for $F=5 \mathrm{kV} / \mathrm{cm}$ ) an admixture of the direct localization occurs (the single contour in the upper right part of the plot) with a small proba- 
bility to find both electron and the hole in the right dot. These cross-admixtures are related to the opening of the avoided crossing between the direct and indirect energy levels (see Fig. 6c).

For stronger field (see the plot for $F=15 \mathrm{kV} / \mathrm{cm}$ in Fig. 7) in the ground state the exciton becomes indirect (and consequently dark - see Fig. 6c) with the hole in the right dot and the electron in the left one. The second and the third energy levels correspond to direct excitons in the left and right dots, respectively. In the fourth state the exciton is indirect and the localization of the particles is opposite to the one occurring in the ground state.

Figure 6d shows the spectrum for the same parameters as in Fig. 6c but with the electron-hole interaction switched-off. No avoided crossing is observed for nonzero $F$. At $F=0$ one observes double degeneracy of the ground state and the first excited state, like in the interacting case of Fig. 6c. However, for the non-interacting case the energy splitting between the ground state and the first excited state is only due to the electron tunnel coupling and not to the interaction. The splitting is therefore much smaller than in the interacting case.

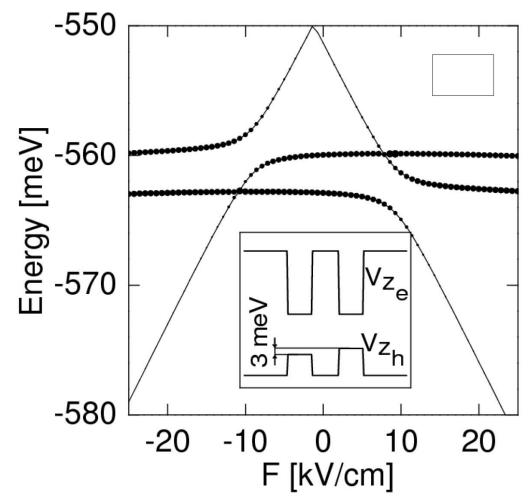

Fig. 8. The same as Fig. 6c but for the left dot shallower for the hole by $3 \mathrm{meV}$. Reprinted figure with permission from Ref. [7]. Copyright (2005) by the American Physical Society.

We are now only a step from the exciton dissociation as observed in the experiment. To make this step let us assume that the dots are not exactly identical. Figure 8 shows the spectrum for the left dot shallower for the hole by $3 \mathrm{meV}$. This is a very small difference, which was assumed to contain both avoided crossings at the positive and negative sides of $F=0$ in the same plot. The corresponding electron and hole distribution is plotted in Fig. 9. In the spectrum of Fig. 8 we see two direct exciton energy levels which only weakly depend of $F$ (one near $E=-560 \mathrm{meV}$, and another near $-563 \mathrm{meV}$ ) and which are avoided crossed by energy levels of indirect exciton states near $F= \pm 10 \mathrm{kV} / \mathrm{cm}$. In the ground state at $F=0$ both the particles are localized in the right dot (see Fig. 9). In the ground 


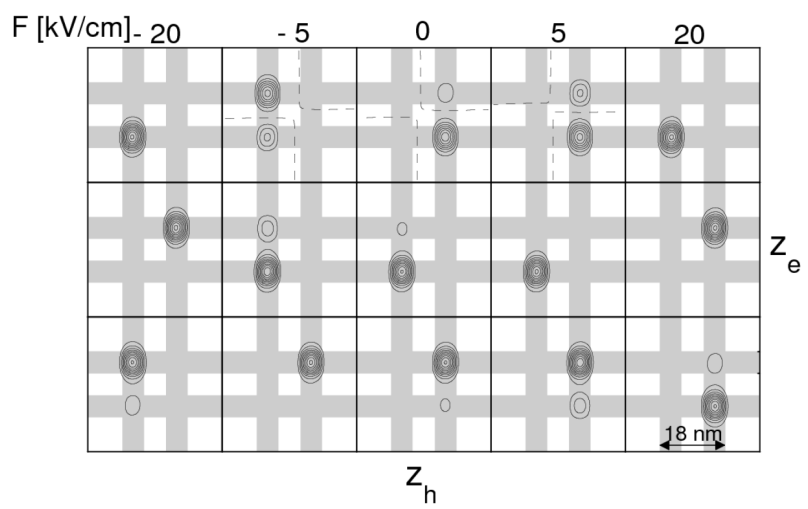

Fig. 9. The same as Fig. 7 for the asymmetric electron-hole confinement of Fig. 8 . Reprinted figure with permission from Ref. [7]. Copyright (2005) by the American Physical Society.

state the exciton dissociation at $F>0$ occurs through removal of the electron from the right dot which is occupied by the hole. The ground-state exciton dissociation at $F<0$ occurs when the hole is removed from the right dot. Since the hole states are not coupled at this value of the barrier thickness the removal of the hole occurs through a very narrow avoided crossing, which at the presented energy scale looks in fact like a crossing of energy levels. For identical dots (see Fig. 6c) we had an energy level which went straight through the center of the avoided crossing. For non-identical dots it is removed off the avoided crossing range to a different energy range - in case of Fig. 8 by about $+3 \mathrm{meV}$.

The ground-state exciton dissociation with a pronounced avoided crossing of spectral lines as shown in Fig. 8 was observed in photoluminescence experiment of Ref. [4]. The experimental work [4] was submitted two months after our paper [7], but it was published first. Our paper [7] contained also a prediction of the mechanism of the negative trion dissociation by the field which was observed [18] over a year after publication of our work. This mechanism is described in the next section.

\section{Dissociation of negative exciton trion by the electric field}

In bulk crystals exciton trions, i.e. charged exciton complexes composed of two holes and a single electron (positive trion $\mathrm{X}^{+}$) or two electrons and a single hole (negative trion $\mathrm{X}^{-}$) are very fragile objects that possess very small binding energies. Their stability is significantly increased in semiconductor quantum wells as compared to the bulk [23]. In bulk crystals as well as in semiconductor quantum wells the binding energy of the trion with respect to dissociation of the complex into an exciton and a free carrier can be identified with the redshift of the photoluminescence line due to the recombination of an electron-hole pair of a trion with respect to the ground-state exciton line. In bulk, quantum wells and wires the 
trion lines in the photoluminescence spectrum need to be redshifted with respect to the exciton line, otherwise the complex is unstable and would not be formed.

The blueshift of the trion lines with respect to the exciton can only be observed in a quantum dot. The problem of the trion binding becomes irrelevant in a quantum dot. The electrons and holes are confined by the quantum dot potential and no trion dissociation is possible, unless a carrier is totally removed of the quantum dot, which typically requires an energy of the order of $100 \mathrm{meV}$. We studied the positions of the trion lines with respect to the exciton line in quantum dots in Refs. [9, 8] using an extensive variational calculations with basis of correlated Gaussians and a generalization of Hamiltonian (1) to the three-particle complex. The shift of the negative trion is defined as

$$
\Delta S\left(\mathrm{X}^{-}\right)=E(\mathrm{X})-\left[E\left(\mathrm{X}^{-}\right)-E_{\mathrm{e}}\right] .
$$

The value in the square brackets gives the energy of the photon appearing at the recombination of an electron-hole pair originally forming the trion. $E_{\mathrm{e}}$ is the final state energy after the electron-hole recombination i.e. the ground-state energy of the electron which remains in the dot. $E\left(\mathrm{X}^{-}\right)$is the ground state energy of the trion (the energy of the initial state). The equivalent expression for the positive trion shift is given by

$$
\Delta S\left(\mathrm{X}^{+}\right)=E(\mathrm{X})-\left[E\left(\mathrm{X}^{+}\right)-E_{\mathrm{h}}\right] .
$$

Positive value of $\Delta S$ corresponds to the redshift and negative - to the blueshift.

We found for both spherical [9] and cylindrical dots [8] that the negative trion is always redshifted with respect to the exciton line. The positive trion line becomes blueshifted with respect to the exciton for small dots. Shifts for the cylindrical dot of height $2 Z$ and diameter $2 R$ are plotted [8] in Fig. 10. Curves are plotted for various $Z$ in function of $R$. For a cylindrical quantum dot with diameter of about $24 \mathrm{~nm}$ and height of about $4 \mathrm{~nm}$ (the values corresponding to the size of a self-assembled dot) we found the redshift of $\mathrm{X}^{-}$of about $7 \mathrm{meV}$, and the blue shift of $\mathrm{X}^{+}$by about $2.5 \mathrm{meV}$ (see the black circles in Fig. 10). Calculations of Fig. 10 [8] were performed before measurements of both negative and positive trion lines coming from the same single dot were accessible. The obtained values [8] well correspond to the ones observed in recent experiments on self-assembled dots - see Fig. 2 of Ref. [5] (left part for a single dot) for instance.

According to the interpretation we proposed in Ref. [9] the character of the shift (i.e. the color - blue or red) that we found by variational calculations can be explained as due to a difference in localization of electrons and holes in quantum dots. In the strong confinement limit (small $R$ and $Z$ ), where the shifts are most pronounced - see Fig. 10 - one can use an approximation of frozen single-particle wave functions (frozen means here: not affected by the interaction). With this assumption the interaction between the particles can be introduced by the first-order perturbation theory. Accordingly, the negative trion energy is $E\left(\mathrm{X}^{-}\right)=2 E_{\mathrm{e}}+E_{\mathrm{h}}-2 V_{\mathrm{eh}}+V_{\mathrm{ee}}$ and the exciton energy writes $E(\mathrm{X})=E_{\mathrm{e}}+E_{\mathrm{h}}-V_{\mathrm{eh}}$, 


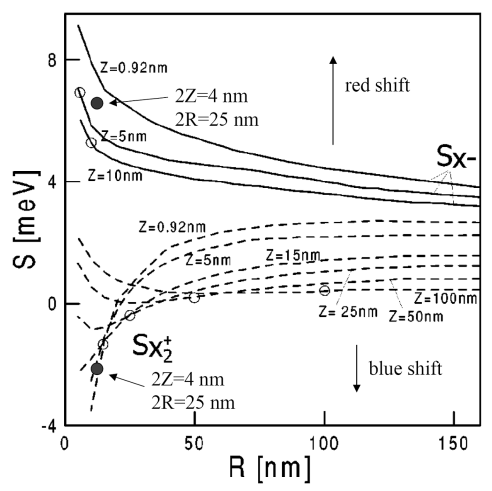

Fig. 10. Shifts of the negative (solid lines) and positive (dashed lines) exciton trion recombination lines with respect to the exciton line for a cylindrical dot of height $2 Z$ and diameter $2 R$. Dots marked by arrows correspond to shifts for diameter $25 \mathrm{~nm}$ and height $4 \mathrm{~nm}$ (values corresponding to the self-assembled dots). Reprinted figure with permission from Ref. [8]. Copyright (2002) by the American Physical Society.

where $V_{\mathrm{ee}}$ and $V_{\mathrm{eh}}$ are the absolute values of the electron-electron and electronhole interaction. The interaction energies are calculated as quantum mechanical mean values of the interaction for single-particle wave functions as obtained for neglected interaction. Consequently, the shift of the negative trion becomes

$$
\Delta S\left(\mathrm{X}^{-}\right)=V_{\mathrm{eh}}-V_{\mathrm{ee}}
$$

Let us note that the single-particle effects contributing to the electron and hole energies $E_{\mathrm{e}}$ and $E_{\mathrm{h}}$ cancel and we are left only with the interaction energies. Therefore, the shifts of the trions lines with respect to the exciton are in a single dot due to the interaction and not the single-particle effects. Due to their larger masses holes are more strongly localized within the dots than electrons. Consequently the absolute value of the hole-hole interaction energy $\left(V_{\mathrm{hh}}\right)$ is larger than the electronhole interaction $\left(V_{\mathrm{eh}}\right)$, which in turn is larger than the electron-electron interaction $\left(V_{\mathrm{ee}}\right)$. Hence the redshift of the negative trion $S\left(\mathrm{X}^{-}\right)>0$ and the blueshift of the positive one $S\left(\mathrm{X}^{+}\right)=V_{\mathrm{eh}}-V_{\mathrm{hh}}<0$.

In Ref. [7] we considered a system of two electrons and a single hole in an asymmetric pair of dots with an external electric field. The calculations were performed with the assumption of the frozen lateral confinement. The three-particle Hamiltonian was integrated over the lateral degrees of freedom which produces an effective Hamiltonian dependent on the vertical coordinates of the three particles only,

$$
\begin{aligned}
H_{\mathrm{eff}}^{\mathrm{X}} & =-\frac{\hbar^{2}}{2 m_{\mathrm{e}}}\left(\frac{\partial^{2}}{\partial z_{\mathrm{e} 1}^{2}}+\frac{\partial^{2}}{\partial z_{\mathrm{e} 2}^{2}}\right)-\frac{\hbar^{2}}{2 m_{\mathrm{h}}} \frac{\partial^{2}}{\partial z_{\mathrm{h}}^{2}}+V_{\mathrm{e}}\left(z_{\mathrm{e} 1}\right) \\
& +V_{\mathrm{e}}\left(z_{\mathrm{e} 2}\right)+V_{\mathrm{h}}\left(z_{\mathrm{h}}\right)-V_{\mathrm{eff}}\left(\left|z_{\mathrm{e} 1}-z_{\mathrm{h}}\right|\right)
\end{aligned}
$$




$$
\begin{aligned}
& -V_{\mathrm{eff}}\left(\left|z_{\mathrm{e} 2}-z_{\mathrm{h}}\right|\right)+V_{\mathrm{eff}}^{\mathrm{ee}}\left(\left|z_{\mathrm{e} 2}-z_{\mathrm{e} 1}\right|\right) \\
& +e F\left(z_{\mathrm{e} 1}\right)+e F\left(z_{\mathrm{e} 2}\right)-e F\left(z_{\mathrm{h}}\right),
\end{aligned}
$$

where $z_{\mathrm{e} 1}$ and $z_{\mathrm{e} 2}$ are coordinates of the first and second electron, respectively. In this Hamiltonian $V_{\text {eff }}, V_{\text {eff }}^{\text {ee }}$ are the effective potentials of electron-hole and electronelectron interaction [24] that are obtained from the original Hamiltonian after its integration with the frozen lateral electron and hole wave functions. The groundstate wave function of this Hamiltonian was found with a finite-difference technique on a three-dimensional mesh of points $\left(z_{\mathrm{e} 1}, z_{\mathrm{e} 2}, z_{\mathrm{h}}\right)$. This approach keeps an exact account for the interparticle correlations that occur in the vertical direction.

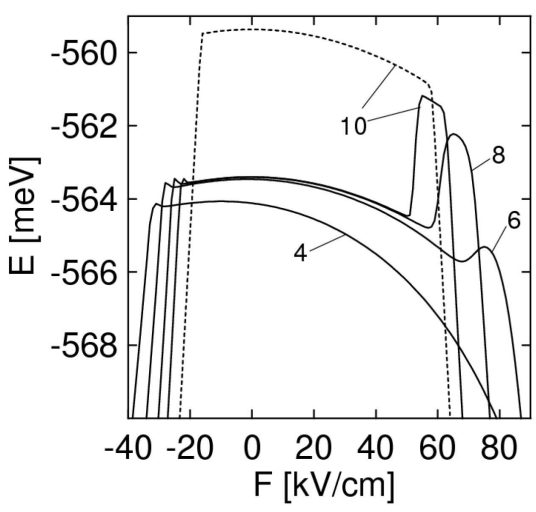

Fig. 11. Solid lines show $E_{\mathrm{t}}=E\left(\mathrm{X}^{-}\right)-E_{\mathrm{e}}$, e.g. the difference of the negative trion ground-state energy and a single-electron ground-state energy in an asymmetric double dot for various barrier thickness (given close to curves in $\mathrm{nm}$ ). Left dot has height of $4 \mathrm{~nm}$ and the right dot has height of $6 \mathrm{~nm}$. The energy difference can be identified with the energy of the trion recombination line in the photoluminescence spectrum calculated with respect to the GaAs energy gap. The dotted line - the exciton ground state energy for $10 \mathrm{~nm}$. Reprinted figure with permission from Ref. [7]. Copyright (2005) by the American Physical Society.

The results are displayed in Fig. 11. The solid curves show $E_{\mathrm{T}}=$ $E\left(\mathrm{X}^{-}\right)-E_{\mathrm{e}}$, i.e. the difference of the trion ground-state energy and a singleelectron ground-state energy - in function of the electric field and for various barrier thickness (which is given close to the curves in $\mathrm{nm}$ ). This energy difference can be identified with the lowest-energy trion line in the photoluminescence spectrum provided that the electrons are not completely separated from the hole (the complete separation occurs at the steep parts of the curves at the left and the right sides of the plot). As everywhere in this paper the energies are given with respect to GaAs energy gap. Let us focus on the plot for the barrier thickness of $10 \mathrm{~nm}$, for which also the exciton ground-state energy (dotted curve) is presented. 
For the field $F \in(-30,50) \mathrm{kV} / \mathrm{cm}$ the trion ground state corresponds to all the three carriers confined in the right dot which is assumed by $2 \mathrm{~nm}$ higher than the left one (heights of the dot are assumed 6 and $4 \mathrm{~nm}$, respectively). In the region of the trion stability the exciton and the trion lines run parallel to one another. The trion line is redshifted by $5 \mathrm{meV}$ and the shift is due to the relative values of the interaction energies between electrons and holes as explained above. For $F<-30 \mathrm{kV} / \mathrm{cm}$ the hole is removed to the left dot which results in the rapid drop of the solid line in Fig. 11. For positive field the hole is stabilized in the right dot but the electrons are removed to the left one. The first electron is removed near $F=50 \mathrm{kV} / \mathrm{cm}$. At the removal the trion line climbs up in the energy reaching nearly the exciton line. The small redshift of the solid line with respect to the exciton line which remains after the removal of the first electron is due to the overall attractive interaction between the electron transferred to the left dot and the electron-hole couple remaining in the right dot. The state with separated electrons remains stable between 50 and $60 \mathrm{kV} / \mathrm{cm}$, and the corresponding recombination energy changes with $F$ in parallel with the exciton energy. For $F>60$ $\mathrm{kV} / \mathrm{cm}$ in the trion ground state the second electron is transferred to the left dot. Let us note that the ground-state exciton dissociation occurs for an electric field which is larger than the field inducing removal of the first electron of the trion but is smaller than the field which removes the second electron.

The discussed features of the trion photoluminescence line with respect to the exciton line were precisely confirmed in the experiment [18] performed several months after our description [7] of the trion dissociation mechanism was published. This, along with the correct picture of the exciton dissociation is quite an achievement for a model which used a very simple confinement potential. The success of the modeling deserves a brief comment. The effect of the electric field on the exciton recombination energy is used to probe the properties of the electron and hole confinement in separate self-assembled quantum dots [25] through deformation of the carrier wave functions. For vertically coupled quantum dots the electric field oriented in the growth direction leads to a redistribution of the carriers between the dots. Typical electric field applied for vertically coupled quantum dots is much smaller than the one used to probe the confinement potential [25] of a single dot (see the range of the electric field of $500 \mathrm{kV} / \mathrm{cm}$ used in Fig. 3 of Ref. [25] to probe a single dot and the scale of $40 \mathrm{kV} / \mathrm{cm}$ used in Fig. 2 of Ref. [5] in a study of a double dot). Therefore experiments on the electric-field effect for coupled dots $[4,5,17,18]$ resolve the properties of the molecular coupling rather than the fabrication dependent details of the confinement in separate dots. Moreover, the charge redistribution induced by the electric field between the different dots produce a much stronger dipole moment than the deformation of the wave functions inside each of the dots (see the slope of the spectral lines as functions of the electric field in Fig. 2 of Ref. [5] in the left part for a single dot and in the right part for the double dot). Last, but not least: the experiments $[4,5,17,18]$ were performed in 
the intermediate coupling regimes when only the electron tunnel coupling between the dots appears. The hole is not transferred between the dots by the field. The spin-orbit coupling for the hole may influence more strongly the spectral features involving the hole interdot transfer for more strongly coupled dots [26].

\section{Non-perfectly aligned quantum dots and a horizontal field orientation}

Vertically stacked quantum dots are aligned one above the other but usually the alignment is not perfect and horizontal shifts of the centers of the dots may be seen in the micrographs of the samples (see Ref. [3] for instance). We recently studied [10] the consequences of the horizontal shifts between the axes of the dots on the exciton photoluminescence spectra. We used three-dimensional quantum well model of the dots with diameter of $20 \mathrm{~nm}$ and height of $4 \mathrm{~nm}$ after the experimental work [4]. The configuration interaction method was used with the single-particle wave functions obtained in a multicenter basis. One of the dots (the one corresponding to negative value of the $z$ coordinate) was assumed deeper by $10 \mathrm{meV}$ for both the electron and the hole. In Fig. 12 we compare the spectrum of ideally aligned dots (a) with a case of non-perfect alignment (b) - with the centers of the dots shifted by $5 \mathrm{~nm}$ in the horizontal (parallel to the $x$-axis) direction - for the electric field oriented vertically (parallel to the $z$-axis). In both cases we notice the avoided crossing pattern of the energy levels known from Fig. 8. The non-perfect alignment only reduces the width of the avoided crossing due to reduced electron tunneling between the dots.

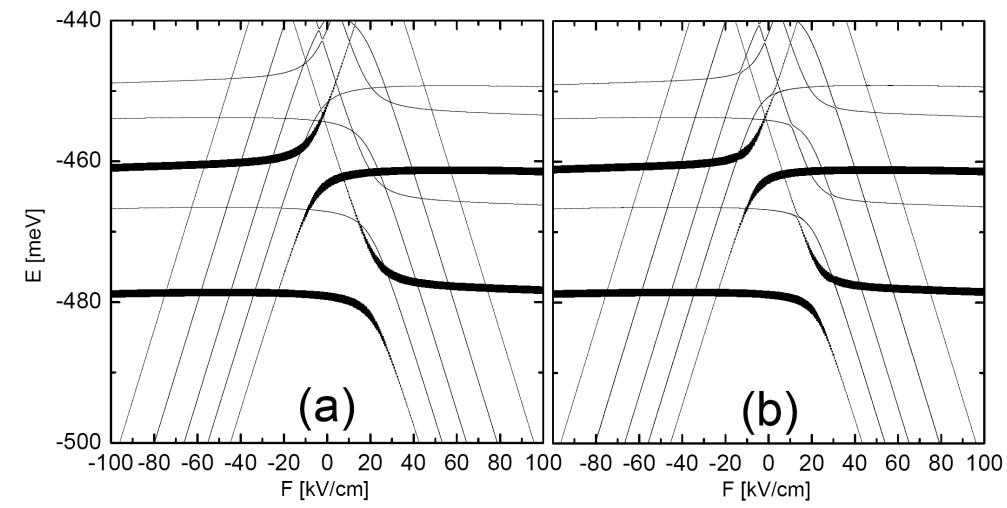

Fig. 12. The exciton spectra for the electric field oriented along the $z$ direction for barrier thickness of $t=6 \mathrm{~nm}$ for (a) perfectly aligned dots, (b) for horizontal distance between the vertical symmetry axis of the dots $d=5 \mathrm{~nm}$. Thickness of the line is proportional to the recombination probability. Reprinted figure with permission from Ref. [10]. Copyright (2007) by the American Physical Society. 


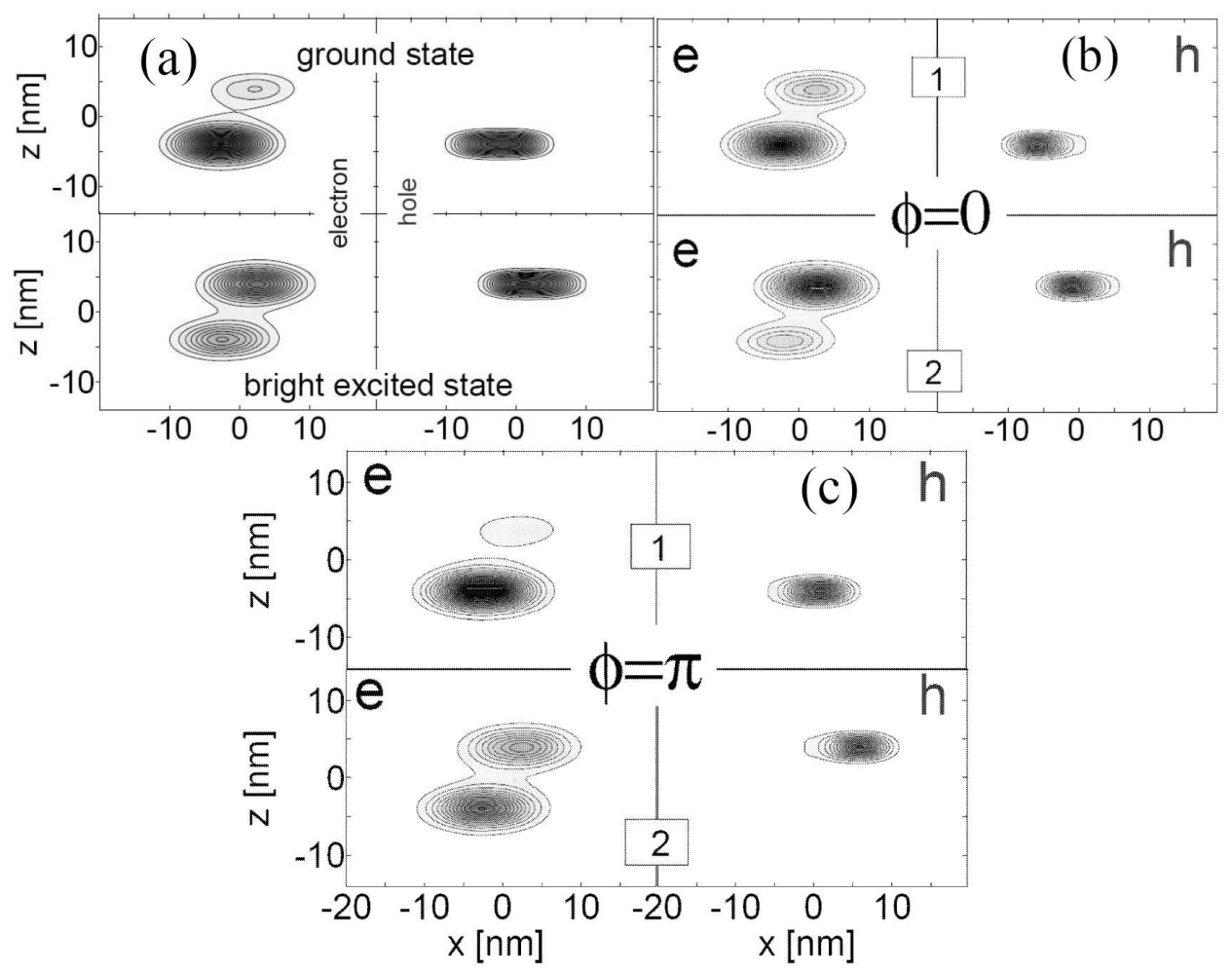

Fig. 13. Electron (left part) and hole (right part) density for a pair of dots separated by barrier $t=4 \mathrm{~nm}$ with horizontal shifts of $5 \mathrm{~nm}$. A cross-section on $y=0$ surface is shown. The centers of dots are located in points $(x, y, z)=( \pm 5 \mathrm{~nm}, 0, \pm 3 \mathrm{~nm})$. The lower dot is deeper by $10 \mathrm{meV}$ for both the electron and the hole. The upper part corresponds to the ground state, and the lower part to the lowest-energy bright excited state. In (a) no electric field is present. In (b) an electric field of $20 \mathrm{kV} / \mathrm{cm}$ is applied in the direction of the $x$ axis (the electric force shifts the electron to the right and the hole to the left). In (c) the orientation of the electric field is inverted with respect to (b). Reprinted figure with permission from Ref. [10]. Copyright (2007) by the American Physical Society.

The electron and hole densities in the absence of the electric field are shown in Fig. 13a for barrier thickness of $4 \mathrm{~nm}$ and a horizontal shift of the dots centers by $5 \mathrm{~nm}$. We display the results for the ground state (upper parts in Fig. 13a,b,c) and the lowest bright energy level (lower parts). In both states the electron is in a molecular orbital, while the hole is in an atomic one. In the ground-state the hole occupies the lower dot (which is deeper) accompanied by most of the electron charge. In the excited state the hole is localized in the upper dot and is also accompanied by most of the electron charge. In the excited state the electron 
tunneling to the other dot is stronger (in the case of the excited state the "other dot" means the "deeper one").

Figure 13b shows the densities for electric field of $20 \mathrm{kV} / \mathrm{cm}$ applied in the horizontal $(x)$ direction. In both the ground state and the excited state we observe an increased electron presence in the right-upper dot. In Fig. 13c the direction of the field is inverted and the electron charge in the left-lower dot is increased. We notice that when $F$ is inverted: the hole distribution within each dot changes, but no hole interdot tunneling occurs. Figure 14a shows the effect of the rotation

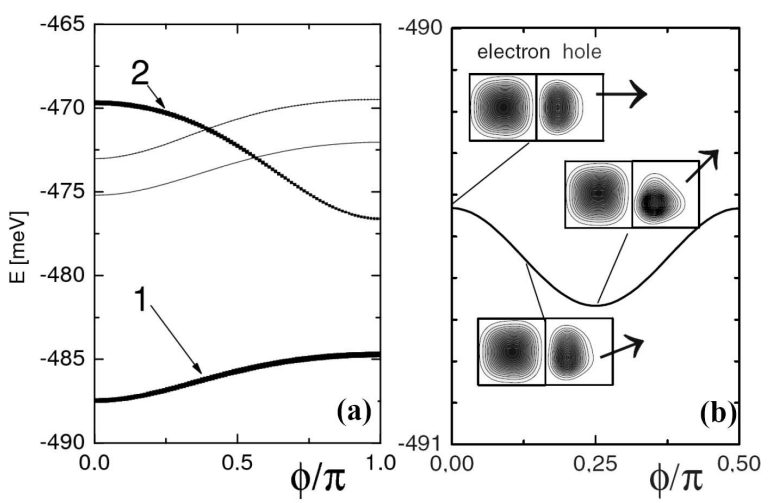

Fig. 14. (a) Exciton spectrum for the pair of dots of Fig. 13 with the electric field vector of $|F|=20 \mathrm{kV} / \mathrm{cm}$ rotated in the horizontal plane $(x, y)$. Thickness of the line is proportional to the recombination probability. Reprinted figure with permission from Ref. [10]. Copyright (2007) by the American Physical Society. (b) The same as in (a) but for the ground-state energy for an exciton in a single square dot. Insets show the electron and hole distribution when the electric field is rotated. The square frames indicate the nominal dot dimensions of $20 \mathrm{~nm}$ side length. Reprinted figure with permission from Ref. [12]. Copyright (2007) by the American Physical Society.

of the field of $20 \mathrm{kV} / \mathrm{cm}$ in the horizontal plane. We see that the extrema of the ground state (marked by "1") and the bright excited state (marked by "2") are antilocked: maximum of " 1 " appears for minimum of " 2 " and vice versa. This can be understood in the following way: when the electric field separates the carriers the exciton ground-state energy becomes lower, and so does the recombination probability. In Fig. 13 we have seen that the maximal separation of the electron and hole occurs in the ground state for $\phi=0$ and in the excited state for $\phi=\pi$, which explains the antilocking phenomenon. According to Fig. 14a one can detect the non-perfect alignment by observation of the exciton spectrum in the rotated electric field. From the exciton spectrum one can also estimate the length and direction of the horizontal shift.

Results of Figs. 12, 13 and 14a were obtained for ideally circular dots. For perfectly aligned circular dots the rotation of an electric field within the plane of 
confinement has no effect on the energy of states (all in-plane $\boldsymbol{F}$ orientations are equivalent). Real dots are not circular, at least not-ideally circular. However, for the weak electric field of $F=20 \mathrm{kV} / \mathrm{cm}$ the details of confinement in separate dots will not be very visible in the spectrum. In Fig. 14b we plotted [12] the ground-state energy for exciton in a single square dot of side length of $20 \mathrm{~nm}$. We see that the energy modulation obtained in the rotated field is of the order of $0.3 \mathrm{meV}$ as compared to $3 \mathrm{meV}$ of Fig. 14a for the system of two ideally circular dots.

\section{Stack of three dots in vertically-oriented electric field}

Recent experiments [16-22] and theoretical papers [27-30] on the electric field effect concerned only artificial molecules formed by a pair of dots. Nevertheless, the growth technique can be applied for production of stacks of multiple dots $[1,2]$. We studied [11] the neutral exciton spectra in stacks formed by three and four dots using a generalization of the configuration interaction approach introduced in Ref. [10].

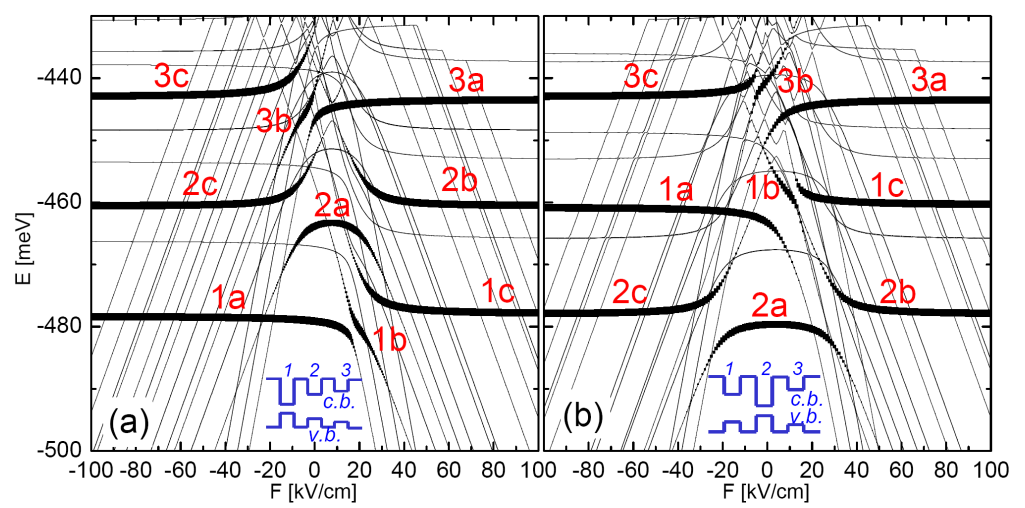

Fig. 15. Exciton energy spectra for a stack of three dots separated by barriers of $6 \mathrm{~nm}$ thickness. In (a) the dot number 1 (see the inset) is deeper by $10 \mathrm{meV}$ than the dot (2) for both the electron and the hole, and the dot 2 is deeper by the same amount than dot 3. In (b) the central dots (number 2) is deeper by $20 \mathrm{meV}$ than the dot number 3 and by $10 \mathrm{meV}$ deeper than the dot number 1 . The thickness of the lines is proportional to the recombination probability. The bright energy levels are labeled by notation composed of an integer 1, 2 or 3 and a letter $a, b$ or $c$. The integer indicates the dot in which the hole is localized, and the letters indicate the states which are bright at $F \ll 0, F \approx 0$ and $F \gg 0$, respectively. Reprinted figure with permission from Ref. [11]. Copyright (2008) by the American Physical Society.

For nominally the same amount of InAs deposited in subsequent layers the dots grown at the top of the stack tend to be larger than the precedent ones. This can be modeled by increasing depth of the confinement potential for both electrons 
and holes along the stack. In Fig. 15a we present the results for a stack of three dots separated by interdot barrier thickness of $t=6 \mathrm{~nm}$. Schematic drawing in the inset shows the increasing depth of the potential. The barrier thickness of $6 \mathrm{~nm}$ corresponds to an intermediate coupling case for which the hole tunnel coupling is not active. Only the electron forms states extended along the stack. We found that in the intermediate coupling the spectrum can be separated into families of energy levels each corresponding to the hole localized in a different dot of the stack. In each family we have $N$ bright energy levels, where $N$ is the number of the dots within the stack. The energy levels of different families cross and avoided crossings are only observed between energy levels of the same family. Moreover, each family possesses its own pattern of avoided crossing.

In the spectrum of Fig. 15a we see three bright energy levels corresponding to each family. Family 1 - composed of $1 \mathrm{a}, 1 \mathrm{~b}$, and $1 \mathrm{c}$ energy levels - is the lowest in the spectrum due to the deepest confinement of the dot number 1 (see the inset). We see that for the family 1 avoided crossings occur only for positive electric field. Positive electric field tends to move the electron to dots of smaller depth (number 2 and 3 in the inset). Avoided crossings occur in the spectrum when the electron localization is interchanged between the $1 \mathrm{a}, 1 \mathrm{~b}$, and $1 \mathrm{c}$ energy levels. Negative electric field only stabilizes the electron in the left dot. In the ground state negative electric field removes the hole to the right dot, but at this barrier thickness the hole transfer is not associated with a pronounced avoided crossing. Let us note that the $1 \mathrm{~b}$ energy level enters the principle avoided crossing of 1a and 1c energy levels and is only temporarily bright - in a finite $F$ interval.

The energy levels of the 3rd family - corresponding to the shallowest dot - are more or less a mirror reflection of the 1st family (see Fig. 15a). In the 2nd family, associated with the central dot, the $2 \mathrm{~b}$ level - bright at $F \approx 0-$ is avoided crossed by $2 \mathrm{a}$ and $2 \mathrm{c}$ energy levels at the negative and the positive side of the origin, respectively. Due to these avoided crossings the $2 \mathrm{~b}$ level in function of the field becomes nearly parabolic.

In Fig. 15b we changed the order of the depths of the dots assuming that the central dot (number 2) is the deepest. We see that the modification of the depths is directly translated into the energy position of each family. The pattern of the avoided crossing of each family remains unaffected. This result indicates that the variation of the confinement potential along the stack can be experimentally detected by observation of the photoluminescence spectrum. For instance one should be able to determine the dot which is the deepest within the stack.

For stronger coupling the hole tunneling is activated which leads to mixing of the energy levels of different families. Figure 16 shows the spectrum for three identical dots spaced by $1 \mathrm{~nm}$ thick barriers. The electron tunnel coupling is extremely strong in this case: spacing between binding and antibinding energy levels is larger than $100 \mathrm{meV}$. The energy spacing of binding and antibinding hole levels is of the order of $10 \mathrm{meV}$. An electric field of $|F| \approx 50 \mathrm{kV} / \mathrm{cm}$ is enough 


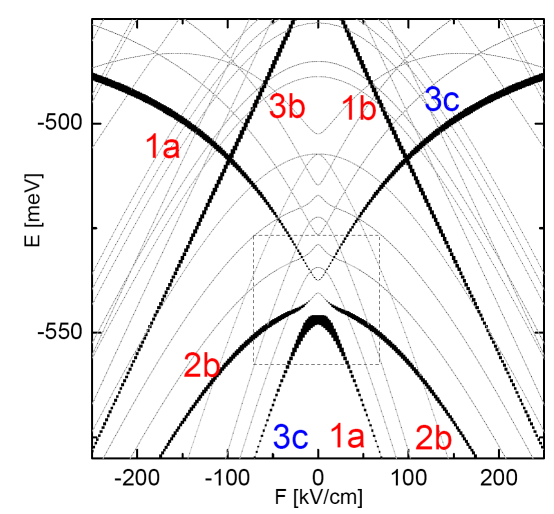

Fig. 16. The same as Fig. 15 but for identical dots spaced by barriers of $1 \mathrm{~nm}$ thickness. Dashed rectangle indicates an avoided crossing between states of different families. Reprinted figure with permission from Ref. [11]. Copyright (2008) by the American Physical Society.

to break the hole tunnel coupling. However, before this happens near $F=0$ we observe avoided crossings of energy levels corresponding to different families. Let us concentrate on the ground-state avoided crossing marked by a rectangle in Fig. 16. Let us note that within the avoided crossing the recombination probability strongly increases in the lowest-energy state at the expense of the two others. In the brightest state the hole distribution becomes compatible with the electron density. The avoided crossing for non-identical dots preserve their character but are shifted to non-zero $F$.

\section{Summary and conclusions}

We discussed the results of the modeling of the interacting electron-hole pair in a vertically stacked InGaAs self-assembled dots. We explained formation of avoided crossings as observed in the photoluminescence spectrum at the exciton ground-state dissociation by the electric field. We discussed the shifts of the trion lines in the photoluminescence spectrum of a single dot as well as the negative trion dissociation by an external electric field applied to a couple of dots. We discussed how the non-perfect alignment of pairs of dots and the potential profile in a stack of self-assembled dots can be probed by the exciton recombination energies in the external electric field.

The modeling was based on simple confinement potentials, single valence band approximation, with neglected electron-hole exchange. With these assumptions the electron-hole correlations can be described exactly. The model correctly predicted qualitative features of the neutral exciton and charged negative trion dissociation by the external field as later observed experimentally. The photoluminescence spectra of neutral and charged excitons in external electric field for 
barrier thicknesses that prevent the hole from tunneling are governed mainly by the effects of the electron interdot tunneling and interaction between the carriers.

\section{Acknowledgments}

The reviewed material comes from papers co-authored by Janusz Adamowski, Stanisław Bednarek, Ewelina Barczyk, and Tomasz Chwiej of AGH Kraków (Poland), by Bart Partoens and François Peeters of the University of Antwerp (Belgium), and by Bernard Stébé of the University of Metz (France).

\section{References}

[1] G.S. Solomon, J.A. Trezza, A.F. Marshall, J.S. Harris Jr., Phys. Rev. Lett. 76, 952 (1996).

[2] N.N. Ledentsov, V.A. Shchukin, M. Grundmann, N. Kirstaedter, J. Böhrer, O. Schmidt, D. Bimberg, V.M. Ustinov, A.Yu. Egorov, A.E. Zhukov, P.S. Kop'ev, S.V. Zaitsev, N.Yu. Gordeev, Zh.I. Alferov, A.I. Borovkov, A.O. Kosogov, S.S. Ruvimov, P. Werner, U. Gösele, J. Heydenreich, Phys. Rev. B 54, 8743 (1996).

[3] S. Fafard, M. Spanner, J.P. McCaffrey, Z.R. Wasilewski, Appl. Phys. Lett. 76, 2268 (2000).

[4] H.J. Krenner, M. Sabahtil, E.C. Clark, A. Kress, D. Schuh, M. Bichler, G. Absteiter, J.J. Finley, Phys. Rev. Lett. 94, 057402 (2005).

[5] E.A. Stinaff, M. Scheibner, A.S. Bracker, I.V. Pomonarev, V.L. Korenev, M.E. Ware, M.F. Doty, T.L. Reinecke, D. Gammon, Science 311, 636 (2005).

[6] B. Szafran, S. Bednarek, J. Adamowski, Phys. Rev. B 64, 125301 (2001).

[7] B. Szafran, T. Chwiej, F.M. Peeters, S. Bednarek, J. Adamowski, B. Partoens, Phys. Rev. B 71, 205316 (2005).

[8] B. Szafran, B. Stébé, S. Bednarek, J. Adamowski, Phys. Rev. B 66, 165331 (2002).

[9] B. Szafran, B. Stébé, S. Bednarek, J. Adamowski, J. Phys., Condens. Matter 12, 2453 (2000).

[10] B. Szafran, F.M. Peeters, S. Bednarek, Phys. Rev. B 75, 115303 (2007).

[11] B. Szafran, E. Barczyk, F.M. Peeters, S. Bednarek, Phys. Rev. B 77, 115441 (2008).

[12] B. Szafran, F.M. Peeters, Phys. Rev. B 76, 195442 (2007).

[13] W. Sheng, J.-P. Leburton, Phys. Rev. Lett. 88, 167401 (2002).

[14] K.L. Janssens, B. Partoens, F.M. Peeters, Phys. Rev. B 65, 233301 (2002).

[15] J.A. Barker, E.P. O'Reilly, Phys. Rev. B 61, 13840 (2000).

[16] G. Ortner, M. Bayer, Y. Lyanda-Geller, T.L. Reinecke, A. Kress, J.P. Reithmaier, A. Forchel, Phys. Rev. Lett. 94, 157401 (2005).

[17] A.S. Bracker, M. Scheibner, M.F. Doty, E.A. Stinaff, I.V. Pomonarev, J.C. Kim, L.J. Whitman, T.L. Reinecke, D. Gammon, Appl. Phys. Lett. 89, 233110 (2006).

[18] H.J. Krenner, E.C. Clark, T. Nakaoka, M. Bichler, C. Scheurer, G. Abstreiter, J.J. Finley, Phys. Rev. Lett. 97, 076403 (2006). 
[19] T. Nakaoka, E.C. Clark, H.J. Krenner, M. Sabathil, M. Bichler, Y. Arakawa, G. Abstreiter, J.J. Finley, Phys. Rev. B 74, R121305 (2006).

[20] M.F. Doty, M. Scheibner, I.V. Ponomarev, E.A. Stinaff, A.S. Bracker, V.L. Korenev, T.L. Reinecke, D. Gammon, Phys. Rev. Lett. 97, 197202 (2006).

[21] M. Scheibner, M.F. Doty, I.V. Ponomarev, A.S. Bracker, E.A. Stinaff, V.L. Korenev, T.L. Reinecke, D. Gammon, Phys. Rev. B 75, 245318 (2007).

[22] M. Scheibner, M. Yakes, A.S. Bracker, I.V. Ponomarev, M.F. Doty, C.S. Hellberg, L.J. Whitman, T.L. Reinecke, D. Gammon, Nature (Phys.) 4, 291 (2008).

[23] B. Stébé, A. Ainane, Superlattices Microstruct. 5, 545 (1989).

[24] S. Bednarek, B. Szafran, T. Chwiej, J. Adamowski, Phys. Rev. B 68, 045328 (2003).

[25] P.W. Fry, I.E. Itskevich, D.J. Mowbray, M.S. Skolnick, J.J. Finley, J.A. Barker, E.P. O'Reilly, L.R. Wilson, I.A. Larkin, P.A. Maksym, M. Hopkinson, M. AlKhafaji, J.P.R. David, A.G. Cullis, G. Hill, J.C. Clark, Phys. Rev. Lett. 84, 733 (2000).

[26] M.F. Doty, J.I. Climente, M. Korkusinski, M. Scheibner, A.S. Bracker, P. Hawrylak, D. Gammon, arxiv:0804.3097.

[27] W. Chu, J.L. Zhu, Appl. Phys. Lett. 89, 053122 (2006).

[28] M.H. Degani, G.A. Farias, P.F. Farinas, Appl. Phys. Lett. 89, 152109 (2006).

[29] M.H. Degani, M.Z. Maialle, Phys. Rev. B 75, 115322 (2007).

[30] D. Xu, N. Zhao, J.-L. Zhu, J. Phys., Condens. Matter 20, 045204 (2008). 